\title{
Arithmetic subderivatives and Leibniz-additive functions
}

\author{
Jorma K. Merikoski ${ }^{a}$, Pentti Haukkanen ${ }^{a}$, Timo Tossavainen ${ }^{b}$ \\ ${ }^{a}$ Faculty of Information Technology and Communication Sciences, \\ FI-33014 Tampere University, Finland \\ jorma.merikoski@tuni.fi, pentti.haukkanen@tuni.fi \\ ${ }^{b}$ Department of Arts, Communication and Education, Lulea University of Technology \\ SE-97187 Lulea, Sweden \\ timo.tossavainen@ltu.se \\ Submitted: June 15, 2018 \\ Accepted: March 25, 2019 \\ Published online: April 13, 2019
}

\begin{abstract}
We introduce the arithmetic subderivative of a positive integer with respect to a non-empty set of primes. This notion generalizes the concepts of the arithmetic derivative and arithmetic partial derivative. In order to generalize these notions a step further, we define that an arithmetic function $f$ is Leibniz-additive if there is a nonzero-valued and completely multiplicative function $h_{f}$ satisfying $f(m n)=f(m) h_{f}(n)+f(n) h_{f}(m)$ for all positive integers $m$ and $n$. We study some basic properties of such functions. For example, we present conditions when an arithmetic function is Leibniz-additive and, generalizing the well-known bounds for the arithmetic derivative, we establish bounds for a Leibniz-additive function.
\end{abstract}

Keywords: arithmetic derivative, Leibniz rule, additivity, multiplicativity

$M S C: 11 \mathrm{~A} 25,11 \mathrm{~A} 05$

\section{Introduction}

We let $\mathbb{P}, \mathbb{Z}_{+}, \mathbb{N}, \mathbb{Z}$, and $\mathbb{Q}$ stand for the set of primes, positive integers, nonnegative integers, integers, and rational numbers, respectively. 
Let $n \in \mathbb{Z}_{+}$. There is a unique sequence $\left(\nu_{p}(n)\right)_{p \in \mathbb{P}}$ of nonnegative integers (with only finitely many positive terms) such that

$$
n=\prod_{p \in \mathbb{P}} p^{\nu_{p}(n)} .
$$

We use this notation throughout.

Let $\emptyset \neq S \subseteq \mathbb{P}$. We define the arithmetic subderivative of $n$ with respect to $S$ as

$$
D_{S}(n)=n_{S}^{\prime}=n \sum_{p \in S} \frac{\nu_{p}(n)}{p} .
$$

In particular, $n_{\mathbb{P}}^{\prime}$ is the arithmetic derivative of $n$, defined by Barbeau [2] and studied further by Ufnarovski and Åhlander [10]. Another well-known special case is $n_{\{p\}}^{\prime}$, the arithmetic partial derivative of $n$ with respect to $p \in \mathbb{P}$, defined by Kovič [7] and studied further by the present authors and Mattila [4, 5].

We define the arithmetic logarithmic subderivative of $n$ with respect to $S$ as

$$
\operatorname{ld}_{S}(n)=\frac{D_{S}(n)}{n}=\sum_{p \in S} \frac{\nu_{p}(n)}{p} .
$$

In particular, $\operatorname{ld}_{\mathbb{P}}(n)$ is the arithmetic logarithmic derivative of $n$. This notion was originally introduced by Ufnarovski and Åhlander [10].

An arithmetic function $g$ is completely additive (or c-additive, for short) if $g(m n)=g(m)+g(n)$ for all $m, n \in \mathbb{Z}_{+}$. It follows from the definition that $g(1)=0$. An arithmetic function $h$ is completely multiplicative (or c-multiplicative, for short) if $h(1)=1$ and $h(m n)=h(m) h(n)$ for all $m, n \in \mathbb{Z}_{+}$. The following theorems recall that these functions are totally determined by their values at primes. The proofs are simple and omitted.

Theorem 1.1. Let $g$ be an arithmetic function, and let $\left(x_{p}\right)_{p \in \mathbb{P}}$ be a sequence of real numbers. The following conditions are equivalent:

(a) $g$ is c-additive and $g(p)=x_{p}$ for all $p \in \mathbb{P}$;

(b) for all $n \in \mathbb{Z}_{+}$,

$$
g(n)=\sum_{p \in \mathbb{P}} \nu_{p}(n) x_{p} .
$$

Theorem 1.2. Let $h$ be an arithmetic and nonzero-valued function, and let $\left(y_{p}\right)_{p \in \mathbb{P}}$ be a sequence of nonzero real numbers. The following conditions are equivalent:

(a) $h$ is c-multiplicative and $h(p)=y_{p}$ for all $p \in \mathbb{P}$;

(b) for all $n \in \mathbb{Z}_{+}$,

$$
h(n)=\prod_{p \in \mathbb{P}} y_{p}^{\nu_{p}(n)}
$$


We say that an arithmetic function $f$ is Leibniz-additive (or L-additive, for short) if there is a nonzero-valued and c-multiplicative function $h_{f}$ such that

$$
f(m n)=f(m) h_{f}(n)+f(n) h_{f}(m)
$$

for all $m, n \in \mathbb{Z}_{+}$. Then $f(1)=0$, since $h_{f}(1)=1$. The property (1.2) may be considered a generalized Leibniz rule. Substituting $m=n=p \in \mathbb{P}$ and applying induction, we get

$$
f\left(p^{a}\right)=a f(p) h(p)^{a-1}
$$

for all $p \in \mathbb{P}, a \in \mathbb{Z}_{+}$.

The arithmetic subderivative $D_{S}$ is L-additive with $h_{D_{S}}=N$, where $N$ is the identity function $N(n)=n$. A c-additive function $g$ is L-additive with $h_{g}=E$, where $E(n)=1$ for all $n \in \mathbb{Z}_{+}$. The arithmetic logarithmic subderivative $\operatorname{ld}_{S}$ is c-additive and hence L-additive.

This paper is a sequel to [6], where we defined L-additivity without requiring that $h_{f}$ is nonzero-valued. We begin by showing how the values of an L-additive function $f$ are determined in $\mathbb{Z}_{+}$by the values of $f$ and $h_{f}$ at primes (Section 2) and then study under which conditions an arithmetic function $f$ can be expressed as $f=g h$, where $g$ is c-additive and $h$ is nonzero-valued and c-multiplicative (Section 3). It turns out that the same conditions are necessary for L-additivity (Section 4). Finally, extending Barbeau's [2] and Westrick's [11] results, we present some lower and upper bounds for an L-additive function (Section 5). We complete our paper with some remarks (Section 6).

\section{Constructing $f(n)$ and $h_{f}(n)$}

An L-additive function $f$ is not totally defined by its values at primes. Also, the values of $h_{f}$ at primes must be known.

Theorem 2.1. Let $f$ be an arithmetic function, and let $\left(x_{p}\right)_{p \in \mathbb{P}}$ and $\left(y_{p}\right)_{p \in \mathbb{P}}$ be as in Theorems 1.1 and 1.2. The following conditions are equivalent:

(a) $f$ is L-additive and $f(p)=x_{p}, h_{f}(p)=y_{p}$ for all $p \in \mathbb{P}$;

(b) for all $n \in \mathbb{Z}_{+}$,

$$
f(n)=\left(\sum_{p \in \mathbb{P}} \nu_{p}(n) \frac{x_{p}}{y_{p}}\right) \prod_{p \in \mathbb{P}} y_{p}^{\nu_{p}(n)} .
$$

Proof. (a) $\Rightarrow(\mathrm{b})$. Since $f(1)=0$, (b) holds for $n=1$. So, let $n>1$. Denoting

$$
\left\{p_{1}, \ldots, p_{s}\right\}=\left\{p \in \mathbb{P} \mid \nu_{p}(n)>0\right\}
$$

and

$$
a_{i}=\nu_{p_{i}}(n), \quad i=1, \ldots, s,
$$


we have

$$
\begin{aligned}
f(n) & =\sum_{i=1}^{s} h_{f}\left(p_{1}\right)^{a_{1}} \cdots h_{f}\left(p_{i-1}\right)^{a_{i-1}} f\left(p_{i}^{a_{i}}\right) h_{f}\left(p_{i+1}\right)^{a_{i+1}} \cdots h_{f}\left(p_{s}\right)^{a_{s}} \\
& =\sum_{i=1}^{s} h_{f}\left(p_{1}\right)^{a_{1}} \cdots h_{f}\left(p_{i-1}\right)^{a_{i-1}} a_{i} f\left(p_{i}\right) h_{f}\left(p_{i}\right)^{a_{i}-1} h_{f}\left(p_{i+1}\right)^{a_{i+1}} \cdots h_{f}\left(p_{s}\right)^{a_{s}} \\
& =\sum_{p \in \mathbb{P}}\left(\nu_{p}(n) f(p) h_{f}(p)^{\nu_{p}(n)-1} \prod_{\substack{q \in \mathbb{P} \\
q \neq p}} h_{f}(q)^{\nu_{q}(n)}\right) \\
& =\sum_{p \in \mathbb{P}}\left(\nu_{p}(n) \frac{f(p)}{h_{f}(p)} \prod_{q \in \mathbb{P}} h_{f}(q)^{\nu_{q}(n)}\right) \\
& =\left(\sum_{p \in \mathbb{P}} \nu_{p}(n) \frac{x_{p}}{y_{p}}\right) \prod_{p \in \mathbb{P}} y_{p}^{\nu_{p}(n)} .
\end{aligned}
$$

The first equation can be proved by induction on $s$, the second holds by (1.3), and the remaining equations are obvious.

(b) $\Rightarrow(\mathrm{a})$. We define now

$$
h(n)=\prod_{p \in \mathbb{P}} y_{p}^{\nu_{p}(n)} .
$$

Let $m, n \in \mathbb{Z}_{+}$. Then

$$
\begin{aligned}
f(m n)= & \left(\sum_{p \in \mathbb{P}} \nu_{p}(m n) \frac{x_{p}}{y_{p}}\right) \prod_{p \in \mathbb{P}} y_{p}^{\nu_{p}(m n)} \\
= & \left(\sum_{p \in \mathbb{P}}\left(\nu_{p}(m)+\nu_{p}(n)\right) \frac{x_{p}}{y_{p}}\right) \prod_{p \in \mathbb{P}} y_{p}^{\nu_{p}(m)+\nu_{p}(n)} \\
= & \left(\sum_{p \in \mathbb{P}}\left(\nu_{p}(m)+\nu_{p}(n)\right) \frac{x_{p}}{y_{p}}\right)\left(\prod_{p \in \mathbb{P}} y_{p}^{\nu_{p}(m)}\right)\left(\prod_{p \in \mathbb{P}} y_{p}^{\nu_{p}(n)}\right) \\
= & \left(\sum_{p \in \mathbb{P}} \nu_{p}(m) \frac{x_{p}}{y_{p}}\left(\prod_{p \in \mathbb{P}} y_{p}^{\nu_{p}(m)}\right)\right)\left(\prod_{p \in \mathbb{P}} y_{p}^{\nu_{p}(n)}\right) \\
& +\left(\sum_{p \in \mathbb{P}} \nu_{p}(n) \frac{x_{p}}{y_{p}}\left(\prod_{p \in \mathbb{P}} y_{p}^{\nu_{p}(n)}\right)\right)\left(\prod_{p \in \mathbb{P}} y_{p}^{\nu_{p}(m)}\right) \\
= & f(m) h(n)+f(n) h(m) .
\end{aligned}
$$

So, $f$ is L-additive with $h_{f}=h$. It is clear that $f(p)=x_{p}$ and $h_{f}(p)=y_{p}$ for all $p \in \mathbb{P}$.

Next, we construct $h_{f}$ from $f$. Let us denote

$$
U_{f}=\{p \in \mathbb{P} \mid f(p) \neq 0\}, \quad V_{f}=\{p \in \mathbb{P} \mid f(p)=0\}
$$


If $f=\theta$, where $\theta(n)=0$ for all $n \in \mathbb{Z}_{+}$, then any $h_{f}$ applies. Hence, we now assume that $f \neq \theta$. Then $U_{f} \neq \emptyset$.

Since

$$
f\left(p^{2}\right)=2 f(p) h_{f}(p)
$$

by (1.3), we have

$$
h_{f}(p)=\frac{f\left(p^{2}\right)}{2 f(p)} \quad \text { for } p \in U_{f}
$$

The case $p \in V_{f}$ remains. Let $q \in \mathbb{P}$. Then (1.2) implies that

$$
f(p q)=f(p) h_{f}(q)+f(q) h_{f}(p)=f(q) h_{f}(p) .
$$

Therefore,

$$
h_{f}(p)=\frac{f(p q)}{f(q)} \quad \text { for } p \in V_{f}
$$

where $q \in U_{f}$ is arbitrary. Now, by Theorem 1.2 ,

$$
h_{f}(n)=\left(\prod_{p \in U_{f}}\left(\frac{f\left(p^{2}\right)}{2 f(p)}\right)^{\nu_{p}(n)}\right)\left(\prod_{p \in V_{f}}\left(\frac{f(p q)}{f(q)}\right)^{\nu_{p}(n)}\right)
$$

where $q \in U_{f}$ is arbitrary. (If $V_{f}=\emptyset$, then the latter factor is the "empty product" one.) We have thus proved the following theorem.

Theorem 2.2. If $f \neq \theta$ is L-additive, then $h_{f}$ is unique and determined by (2.2).

\section{Decomposing $f=g h$}

Let $f$ be an arithmetic function and let $h$ be a nonzero-valued and c-multiplicative function. By Theorem 2.1, $f$ is L-additive with $h_{f}=h$ if and only if

$$
f(n)=\left(\sum_{p \in \mathbb{P}} \nu_{p}(n) \frac{f(p)}{h(p)}\right) \prod_{p \in \mathbb{P}} h(p)^{\nu_{p}(n)}=\left(\sum_{p \in \mathbb{P}} \nu_{p}(n) \frac{f(p)}{h(p)}\right) h(n) .
$$

The function

$$
g(n)=\sum_{p \in \mathbb{P}} \nu_{p}(n) \frac{f(p)}{h(p)}
$$

is c-additive by Theorem 1.1 .

We say that an arithmetic function $f$ is gh-decomposable if it has a gh decomposition

$$
f=g h,
$$

where $g$ is c-additive and $h$ is nonzero-valued and c-multiplicative. We saw above that L-additivity implies $g h$-decomposability. Also, the converse holds. 
Theorem 3.1. Let $f$ be an arithmetic function. The following conditions are equivalent:

(a) $f$ is L-additive;

(b) $f$ is gh-decomposable.

Proof. (a) $\Rightarrow$ (b). We proved this above.

(b) $\Rightarrow$ (a). For all $m, n \in \mathbb{Z}_{+}$,

$$
\begin{aligned}
f(m n) & =g(m n) h(m n)=(g(m)+g(n)) h(m) h(n) \\
& =g(m) h(m) h(n)+g(n) h(n) h(m)=f(m) h(n)+f(n) h(m) .
\end{aligned}
$$

Consequently, $f$ is L-additive with $h_{f}=h$.

Corollary 3.2. Let $f \neq \theta$ be an arithmetic function. The following conditions are equivalent:

(a) $f$ is L-additive;

(b) $f$ is uniquely gh-decomposable.

Proof. In proving (a) $\Rightarrow(\mathrm{b}), h_{f}$ is unique by Theorem 2.2. Since $h_{f}$ is nonzerovalued, also $g=f / h_{f}$ is unique.

For example, if $f=D_{S}$, then $g=\operatorname{ld}_{S}$ and $h=N$.

By Theorem 2.2, an L-additive function $f \neq \theta$ determines $h_{f}$ uniquely. We consider next the converse problem: Given a nonzero-valued and c-multiplicative function $h$, find an L-additive function $f$ such that $h_{f}=h$.

Theorem 3.3. Let $\left(x_{p}\right)_{p \in \mathbb{P}}$ be a sequence of real numbers and let $h$ be nonzerovalued and c-multiplicative. There is a unique L-additive function $f$ with $h_{f}=h$ such that $f(p)=x_{p}$ for all $p \in \mathbb{P}$.

Proof. If at least one $x_{p} \neq 0$, then apply Theorem 2.1 and Corollary 3.2. Otherwise, $f=\theta$.

We can now characterize $D_{S}$ and $\operatorname{ld}_{S}$.

Corollary 3.4. Let $f$ be an arithmetic function and $\emptyset \neq S \subseteq \mathbb{P}$. The following conditions are equivalent:

(a) $f$ is L-additive, $h_{f}=N, f(p)=1$ for $p \in S$, and $f(p)=0$ for $p \in \mathbb{P} \backslash S$;

(b) $f=D_{S}$.

Corollary 3.5. Let $g$ be an arithmetic function and $\emptyset \neq S \subseteq \mathbb{P}$. The following conditions are equivalent:

(a) $g$ is c-additive, $g(p)=1 / p$ for $p \in S$, and $g(p)=0$ for $p \in \mathbb{P} \backslash S$;

(b) $g=\operatorname{ld}_{S}$. 


\section{Conditions for L-additivity}

Let $f \neq \theta$ be L-additive and $a, b \in \mathbb{N}$.

First, let $p \in \mathbb{P}$. By (1.3),

$$
f\left(p^{a+1}\right)=(a+1) f(p) h_{f}(p)^{a}, \quad f\left(p^{b+1}\right)=(b+1) f(p) h_{f}(p)^{b},
$$

and, further,

$$
f\left(p^{a+1}\right)^{b}=(a+1)^{b} f(p)^{b} h_{f}(p)^{a b}, f\left(p^{b+1}\right)^{a}=(b+1)^{a} f(p)^{a} h_{f}(p)^{b a} .
$$

Assume now that $p \in U_{f}$. Then the right-hand sides of the equations in (4.1) are nonzero and $f\left(p^{a+1}\right), f\left(p^{b+1}\right) \neq 0$. Therefore, by (4.2),

$$
\frac{f\left(p^{a+1}\right)^{b}}{f\left(p^{b+1}\right)^{a}}=\frac{(a+1)^{b} f(p)^{b}}{(b+1)^{a} f(p)^{a}}
$$

or, equivalently,

$$
\left(\frac{f\left(p^{a+1}\right)}{(a+1) f(p)}\right)^{b}=\left(\frac{f\left(p^{b+1}\right)}{(b+1) f(p)}\right)^{a} .
$$

Second, assume that $U_{f}$ has at least two elements. If $p, q \in U_{f}$, then (1.2) and (1.3) imply that

$$
\begin{aligned}
f\left(p^{a} q^{b}\right) & =f\left(p^{a}\right) h_{f}\left(q^{b}\right)+f\left(q^{b}\right) h_{f}\left(p^{a}\right) \\
& =f\left(p^{a}\right) h_{f}(q)^{b}+f\left(q^{b}\right) h_{f}(p)^{a}=\frac{f\left(p^{a}\right) f\left(q^{b+1}\right)}{(b+1) f(q)}+\frac{f\left(q^{b}\right) f\left(p^{a+1}\right)}{(a+1) f(p)} .
\end{aligned}
$$

Third, assume additionally that $V_{f} \neq \emptyset$. Let $p \in V_{f}$ and $q_{1}, q_{2} \in U_{f}$. By (2.1) and the fact that $h_{f}$ is nonzero-valued,

$$
\frac{f\left(p q_{1}\right)}{f\left(q_{1}\right)}=\frac{f\left(p q_{2}\right)}{f\left(q_{2}\right)} \neq 0 .
$$

In other words, we can "cancel" $p$ in

$$
\frac{f\left(p q_{1}\right)}{f\left(p q_{2}\right)}=\frac{f\left(q_{1}\right)}{f\left(q_{2}\right)} \neq 0 .
$$

Fourth, both the nonzero-valuedness of $h_{f}$ and (2.2) imply that

$$
f\left(p^{2}\right) \neq 0 \quad \text { for all } p \in U_{f}
$$

We have thus found necessary conditions for L-additivity.

Theorem 4.1. Let $f \neq \theta$ be L-additive and $a, b \in \mathbb{N}$.

(i) If $p \in U_{f}$, then

$$
\left(\frac{f\left(p^{a+1}\right)}{(a+1) f(p)}\right)^{b}=\left(\frac{f\left(p^{b+1}\right)}{(b+1) f(p)}\right)^{a} .
$$


(ii) If $p, q \in U_{f}$, then

$$
f\left(p^{a} q^{b}\right)=\frac{f\left(p^{a}\right) f\left(q^{b+1}\right)}{(b+1) f(q)}+\frac{f\left(q^{b}\right) f\left(p^{a+1}\right)}{(a+1) f(p)} .
$$

(iii) If $p \in V_{f}$ and $q_{1}, q_{2} \in U_{f}$, then

$$
\frac{f\left(p q_{1}\right)}{f\left(p q_{2}\right)}=\frac{f\left(q_{1}\right)}{f\left(q_{2}\right)} \neq 0 .
$$

(iv) If $p \in U_{f}$, then

$$
f\left(p^{2}\right) \neq 0
$$

The question about the sufficiency of these conditions remains open.

To find sufficient conditions for L-additivity, we study under which conditions we can apply the procedure described in the proof of Theorem 2.2 to a given arithmetic function $f \neq \theta$. The function $h$, defined as $h_{f}$ in $(2.2)$, must be $(\alpha)$ well-defined, $(\beta)$ c-multiplicative, and $(\gamma)$ nonzero-valued. Condition $(\alpha)$ follows from (iii), ( $\beta$ ) is obvious, and $(\gamma)$ follows from (iii) and (iv). If the function $g=f / h$ is also c-additive, then $f$ is L-additive by Theorem 3.1. So, we have found sufficient conditions for L-additivity, and they are obviously also necessary.

Theorem 4.2. An arithmetic function $f \neq \theta$ is L-additive if and only if (iii) and (iv) in Theorem 4.1 are satisfied and the function $f / h$ is c-additive, where

$$
h(n)=\left(\prod_{p \in U_{f}}\left(\frac{f\left(p^{2}\right)}{2 f(p)}\right)^{\nu_{p}(n)}\right)\left(\prod_{p \in V_{f}}\left(\frac{f(p q)}{f(q)}\right)^{\nu_{p}(n)}\right), \quad q \in U_{f} .
$$

\section{Bounds for an L-additive function}

Let us express (1.1) as

$$
n=q_{1} \cdots q_{r}
$$

where $q_{1}, \ldots, q_{r} \in \mathbb{P}, q_{1} \leq \cdots \leq q_{r}$. We first recall the well-known bounds for $D(n)$ using $n$ and $r$ only.

Theorem 5.1. Let $n$ be as in (5.1). Then

$$
r n^{\frac{r-1}{r}} \leq D(n) \leq \frac{r n}{2} \leq \frac{n \log _{2} n}{2} .
$$

Equality is attained in the upper bounds if and only if $n$ is a power of 2, and in the lower bound if and only if $n$ is a prime or a power of 2 .

Proof. See [2, pp. 118-119], [10, Theorem 9].

The first upper bound can be improved using the same information. Westrick [11, Ineq. (6)] presented in her thesis the following bound without proof. 
Theorem 5.2. Let $n$ be as in (5.1). Then

$$
D(n) \leq \frac{r-1}{2} n+2^{r-1}
$$

Equality is attained if and only if $n \in \mathbb{P}$ or $q_{1}=\cdots=q_{r-1}=2$.

Proof. If $r=1$ (i.e., $n \in \mathbb{P}$ ), then (5.3) clearly holds with equality. So, assume that $r>1$.

Case 1. $q_{1}=\cdots=q_{r-1}=2$. Then

$$
D(n)=n\left(\frac{r-1}{2}+\frac{1}{q_{r}}\right)=\frac{r-1}{2} n+\frac{n}{n / 2^{r-1}}=\operatorname{rhs}(5.3),
$$

where "rhs" is short for "the right-hand side".

Case 2. $q_{1}=\cdots=q_{r-2}=2$ (omit this if $r=2$ ) and $q_{r-1}>2$. Since

$$
\frac{1}{q_{r-1}}+\frac{1}{q_{r}}=\frac{1}{2}+\frac{4-\left(q_{r-1}-2\right)\left(q_{r}-2\right)}{2 q_{r-1} q_{r}}<\frac{1}{2}+\frac{2}{q_{r-1} q_{r}},
$$

we have

$$
D(n)<n\left(\frac{r-2}{2}+\frac{1}{2}+\frac{2}{q_{r-1} q_{r}}\right)=\frac{r-1}{2} n+\frac{2 n}{n / 2^{r-2}}=\operatorname{rhs}(5.3) .
$$

Case 3. $q_{r-2}>2$. Then $r \geq 3$ and

$$
D(n) \leq n\left(\frac{r-3}{2}+\frac{1}{3}+\frac{1}{3}+\frac{1}{3}\right)=\frac{r-1}{2} n<\operatorname{rhs}(5.3) .
$$

The claim with equality conditions is thus verified. Because

$$
\frac{r n}{2}-\left(\frac{r-1}{2} n+2^{r-1}\right)=\frac{n}{2}-2^{r-1} \geq \frac{2^{r}}{2}-2^{r-1}=0,
$$

the upper bound (5.3) indeed improves (5.2).

We extend the upper bounds (5.2) and (5.3) under the assumption

$$
h_{f}(p) \geq p \text { for all } p \in U_{f} .
$$

Let $n$ in (5.1) have $q_{i_{1}}, \ldots, q_{i_{s}} \in U_{f}$. We denote

$$
p_{1}=q_{i_{1}}, \ldots, p_{s}=q_{i_{s}}
$$

and

$$
M=\max _{1 \leq i \leq r} f\left(q_{i}\right)=\max _{1 \leq i \leq s} f\left(p_{i}\right) .
$$

Theorem 5.3. Let $f \neq \theta$ be nonnegative and L-additive satisfying (5.4). Then

$$
f(n) \leq \frac{s M}{2} h_{f}(n) \leq \frac{M \log _{2} n}{2} h_{f}(n),
$$

where $s$ is as in (5.5) and $M$ is as in (5.6). Equality is attained if and only if $n$ is a power of 2 . 
Proof. By (3.1) and simple manipulation,

$$
\begin{aligned}
f(n) & =h_{f}(n) \sum_{i=1}^{r} \frac{f\left(q_{i}\right)}{h_{f}\left(q_{i}\right)}=h_{f}(n) \sum_{i=1}^{s} \frac{f\left(p_{i}\right)}{h_{f}\left(p_{i}\right)} \leq h_{f}(n) M \sum_{i=1}^{s} \frac{1}{p_{i}} \\
& \leq h_{f}(n) M \sum_{i=1}^{s} \frac{1}{2}=h_{f}(n) M \frac{s}{2} \leq h_{f}(n) M \frac{r}{2} \leq h_{f}(n) M \frac{\log _{2} n}{2} .
\end{aligned}
$$

The equality condition is obvious.

Theorem 5.4. Let $f \neq \theta$ be nonnegative and L-additive satisfying (5.4). Then

$$
f(n) \leq\left(\frac{s-1}{2} h_{f}(n)+h_{f}\left(2^{s-1}\right)\right) M
$$

where $s$ is as in (5.5) and $M$ is as in (5.6). Equality is attained if and only if $n \in \mathbb{P}$ or $p_{1}=\cdots=p_{s-1}=2=h_{f}(2)$.

Proof. If $s=1$ (i.e., $n \in \mathbb{P}$ ), then (5.8) clearly holds with equality. So, assume that $s>1$.

Case 1. $p_{1}=\cdots=p_{s-1}=2$. Then

$$
\begin{aligned}
f(n) & =f\left(2^{s-1} p_{s}\right)=f\left(2^{s-1}\right) h_{f}\left(p_{s}\right)+f\left(p_{s}\right) h_{f}\left(2^{s-1}\right) \\
& =(s-1) f(2) h_{f}\left(2^{s-2}\right) h_{f}\left(p_{s}\right)+f\left(p_{s}\right) h_{f}\left(2^{s-1}\right) \\
& \leq\left((s-1)\left(h_{f}\left(2^{s-2}\right) h_{f}\left(p_{s}\right)+h_{f}\left(2^{s-1}\right)\right) M\right. \\
& \leq\left((s-1) h_{f}\left(2^{s-2}\right) h_{f}\left(p_{s}\right) \frac{h_{f}(2)}{2}+h_{f}\left(2^{s-1}\right)\right) M \\
& =\left(\frac{s-1}{2} h_{f}(n)+h_{f}\left(2^{s-1}\right)\right) M .
\end{aligned}
$$

Case 2. $p_{1}=\cdots=p_{s-2}=2$ (omit this if $s=2$ ) and $p_{s-1}>2$. If $s \geq 3$, then

$$
\begin{aligned}
f(n) & =f\left(2^{s-2} p_{s-1} p_{s}\right)=f\left(2^{s-2}\right) h_{f}\left(p_{s-1} p_{s}\right)+f\left(p_{s-1} p_{s}\right) h_{f}\left(2^{s-2}\right) \\
& =(s-2) f(2) h_{f}\left(2^{s-3}\right) h_{f}\left(p_{s-1} p_{s}\right)+f\left(p_{s-1} p_{s}\right) h_{f}\left(2^{s-2}\right) \\
& =\frac{s-2}{2} f(2) h_{f}\left(2^{s-2}\right) h_{f}\left(p_{s-1} p_{s}\right)+\left(f\left(p_{s-1}\right) h_{f}\left(p_{s}\right)+f\left(p_{s}\right) h_{f}\left(p_{s-1}\right)\right) h_{f}\left(2^{s-2}\right) \\
& \leq\left(\frac{s-2}{2} h_{f}\left(2^{s-2}\right) h_{f}\left(p_{s-1} p_{s}\right)+\left(h_{f}\left(p_{s-1}\right)+h_{f}\left(p_{s}\right)\right) h_{f}\left(2^{s-2}\right)\right) M \\
& =\left(\frac{s-2}{2} h_{f}(n)+\left(h_{f}\left(p_{s-1}\right)+h_{f}\left(p_{s}\right)\right) h_{f}\left(2^{s-2}\right)\right) M \\
& =\left(\frac{s-1}{2} h_{f}(n)+\left(h_{f}\left(p_{s-1}\right)+h_{f}\left(p_{s}\right)\right) h_{f}\left(2^{s-2}\right)-\frac{1}{2} h_{f}(n)\right) M .
\end{aligned}
$$

The last expression is obviously an upper bound for $f(n)$ also if $s=2$. If

$$
\left(h_{f}\left(p_{s-1}\right)+h_{f}\left(p_{s}\right)\right) h_{f}\left(2^{s-2}\right)-\frac{1}{2} h_{f}(n) \leq h_{f}\left(2^{s-1}\right),
$$


i.e.,

$$
2\left(h_{f}\left(p_{s-1}\right)+h_{f}\left(p_{s}\right)\right)-h_{f}\left(p_{s-1}\right) h_{f}\left(p_{s}\right) \leq 2 h_{f}(2),
$$

then (5.8) follows. Since

$$
\begin{aligned}
h_{f}\left(p_{s-1}\right) h_{f}\left(p_{s}\right)-2\left(h_{f}\left(p_{s-1}\right)+h_{f}\left(p_{s}\right)\right)+4 & =\left(h_{f}\left(p_{s-1}\right)-2\right)\left(h_{f}\left(p_{s}\right)-2\right) \\
& \geq\left(p_{s-1}-2\right)\left(p_{s}-2\right)>0,
\end{aligned}
$$

we actually have a stronger inequality

$$
2\left(h_{f}\left(p_{s-1}\right)+h_{f}\left(p_{s}\right)\right)-h_{f}\left(p_{s-1}\right) h_{f}\left(p_{s}\right)<4 .
$$

Case 3. $p_{s-2}>2$. Then $s \geq 3$ and

$$
\begin{aligned}
f(n) & =f\left(p_{1}\right) h_{f}\left(p_{2} \cdots p_{s}\right)+f\left(p_{2} \cdots p_{s}\right) h_{f}\left(p_{1}\right) \\
& =f\left(p_{1}\right) \frac{h_{f}(n)}{h_{f}\left(p_{1}\right)}+f\left(p_{2} \cdots p_{s}\right) h_{f}\left(p_{1}\right) \\
& \leq \frac{M h_{f}(n)}{2}+f\left(p_{2} \cdots p_{s}\right) h_{f}\left(p_{1}\right) .
\end{aligned}
$$

Since

$$
\begin{aligned}
f\left(p_{2} \cdots p_{s}\right) h_{f}\left(p_{1}\right) & =\left(f\left(p_{2}\right) h_{f}\left(p_{3} \cdots p_{s}\right)+f\left(p_{3} \cdots p_{s}\right) h_{f}\left(p_{2}\right)\right) h_{f}\left(p_{1}\right) \\
& =f\left(p_{2}\right) \frac{h_{f}(n)}{h_{f}\left(p_{2}\right)}+f\left(p_{3} \cdots p_{s}\right) h_{f}\left(p_{1} p_{2}\right) \\
& \leq \frac{M h_{f}(n)}{2}+f\left(p_{3} \cdots p_{s}\right) h_{f}\left(p_{1} p_{2}\right)
\end{aligned}
$$

we also have

$$
f(n) \leq 2 \frac{M h_{f}(n)}{2}+f\left(p_{3} \cdots p_{s}\right) h_{f}\left(p_{1} p_{2}\right)
$$

Similarly,

$$
f(n) \leq \frac{s-3}{2} M h_{f}(n)+f\left(p_{s-2} p_{s-1} p_{s}\right) h_{f}\left(p_{1} \cdots p_{s-3}\right) .
$$

Because

$$
\begin{aligned}
f\left(p_{s-2} p_{s-1} p_{s}\right) & =f\left(p_{s-2}\right) h_{f}\left(p_{s-1} p_{s}\right)+f\left(p_{s-1}\right) h_{f}\left(p_{s-2} p_{s}\right)+f\left(p_{s}\right) h_{f}\left(p_{s-2} p_{s-1}\right) \\
& \leq M h_{f}\left(p_{s-2} p_{s-1} p_{s}\right)\left(\frac{1}{p_{s-2}}+\frac{1}{p_{s-1}}+\frac{1}{p_{s}}\right) \\
& \leq M h_{f}\left(p_{s-2} p_{s-1} p_{s}\right)\left(\frac{1}{3}+\frac{1}{3}+\frac{1}{3}\right)=M h_{f}\left(p_{s-2} p_{s-1} p_{s}\right),
\end{aligned}
$$

it follows from (5.9) that

$$
f(n) \leq \frac{s-3}{2} M h_{f}(n)+M h_{f}(n)=\frac{s-1}{2} M h_{f}(n) .
$$

In other words, (5.8) holds strictly.

The proof is complete. It also includes the equality conditions. 
If we do not know $s$ (but know $r$ ), we can substitute $s=r$ in (5.7) and (5.8). We complete this section by extending the lower bound (5.2).

Theorem 5.5. Let $f$ be nonnegative and L-additive, and let $n$ be as in (5.1) with

$$
h_{f}\left(q_{1}\right), \ldots, h_{f}\left(q_{r}\right)>0 .
$$

Then

$$
f(n) \geq r m h_{f}(n)^{\frac{r-1}{r}}
$$

where

$$
m=\min _{1 \leq i \leq r} f\left(q_{i}\right) .
$$

Equality is attained if and only if $n$ is a prime or a power of 2 .

Proof. By (3.1) and the arithmetic-geometric mean inequality,

$$
\begin{aligned}
f(n) & =h_{f}(n) \sum_{i=1}^{r} \frac{f\left(q_{i}\right)}{h_{f}\left(q_{i}\right)} \geq h_{f}(n) m \sum_{i=1}^{r} \frac{1}{h_{f}\left(q_{i}\right)} \geq h_{f}(n) m \frac{r}{\left(h_{f}\left(q_{1}\right) \cdots h_{f}\left(q_{r}\right)\right)^{\frac{1}{r}}} \\
& =h_{f}(n) m \frac{r}{h_{f}\left(q_{1} \cdots q_{r}\right)^{\frac{1}{r}}}=h_{f}(n) m \frac{r}{h_{f}(n)^{\frac{1}{r}}}=r h_{f}(n)^{1-\frac{1}{r}} m .
\end{aligned}
$$

The equality condition is obvious.

\section{Concluding remarks}

According to the common custom, we credited in Section 1 the arithmetic derivative to Barbeau [2]. However, Mingot Shelly [8] considered it as early as in 1911. His paper has been overlooked for a long time and is found only recently $[1,9]$. The only reference to it that we know from the past decades is in Dickson [3].

A nice introduction to the arithmetic derivative is Balzarotti and Lava [1] (written in Italian, but an English reader understands its formulas and mathematical terms). There is an extensive literature about this topic, but much work is still left to be done. For example, there is only a few results about "arithmetic integration" and, more generally, about "arithmetic differential equations".

For another example, let us define $D=D_{\mathbb{P}}$ as a function $\mathbb{Q} \rightarrow \mathbb{Q}$ by allowing $\nu_{p}(n) \in \mathbb{Z}$ in (1.1). What do we know about this function? Not much. We are currently investigating whether $D$ (and, more generally, $D_{S}$ ) is discontinuous everywhere and, if so, how strongly.

The arithmetic partial derivative $D_{p}=D_{\{p\}}$ has received less attention than $D$ and, according to our knowledge, the arithmetic subderivative $D_{S}$ is a new concept. An overall question related to this notion is: Which properties of $D$ and $D_{p}$ can in some way be extended to $D_{S}$ ? Probably the cases of finite $S$ and infinite $S$ must then be studied separately.

As an extension of $D_{S}$, we defined the concept of an L-additive function $f$. For simplicity, we stated (contrary to [6]) that $h_{f}$ must be nonzero-valued. If we allow 
$h_{f}$ to be zero, it turns out that we only meet extra work without gaining anything significant in results. Anyway, a very general question arises: Which properties of $D_{S}$ can be extended to $f$ ? In Section 5 , we found the generalizations of the classical upper and lower bounds of $D$. But what about other properties? This remains to be seen.

\section{References}

[1] G. Balzarotti, P. P. Lava: La derivata aritmetica: Alla scoperta di un nuovo approccio alla teoria dei numeri, Milan: Hoepli, 2013.

[2] E. J. Barbeau: Remarks on an arithmetic derivative, Canadian Mathematical Bulletin 4 (1961), pp. 117-122, DOI: 10.4153/cmb-1961-013-0.

[3] L. E. Dickson: History of the Theory of Numbers, Washington: Carnegie Institution, 1919.

[4] P. Haukkanen, J. K. Merikoski, M. Mattila, T. Tossavainen: The arithmetic Jacobian matrix and determinant, Journal of Integer Sequences 20 (2017), Art. 17.9.2.

[5] P. Haukkanen, J. K. Merikoski, T. Tossavainen: On arithmetic partial differential equations, Journal of Integer Sequences 19 (2016), Art. 16.8.6.

[6] P. Haukkanen, J. K. Merikoski, T. Tossavainen: The arithmetic derivative and Leibnizadditive functions, Notes on Number Theory and Discrete Mathematics 24.3 (2018), pp. 6876, DOI: $10.7546 / \mathrm{nntdm} .2018 .24 .3 .68-76$.

[7] J. Kovič: The arithmetic derivative and antiderivative, Journal of Integer Sequences 15 (2012), Art. 12.3.8.

[8] J. M. Shelly: Una cuestión de la teoría de los números, Asociación española, Granada (1911), pp. 1-12.

[9] N. J. A. Sloane: The On-Line Encyclopedia of Integer Sequences, Seq. A003415.

[10] V. Ufnarovski, B. Anlander: How to differentiate a number, Journal of Integer Sequences 6 (2003), Art. 03.3.4.

[11] L. Westrick: Investigations of the number derivative, Student thesis, Massachusetts Institute of Technology, 2003. 